\title{
Avaliação dos benefícios da coleta de lixo em Palmas, Tocantins: uma aplicação do método de avaliação contingente
}

\section{Evaluation of the garbage collection benefits in Palmas, Tocantins: an application of contingent evaluation method}

\author{
Wilians dos Santos Silva \\ Graduado em Ciências Econômicas pela Universidade Federal do Tocantins (UFT). \\ Mestrando do Programa de Pós-Graduação em Desenvolvimento Regional da UFT
}

\section{Adriano Nascimento da Paixão}

Graduação em Ciências Econômicas. Mestre em Economia pela Universidade Federal da Paraíba (UFPB). Doutor em Economia Aplicada pela Universidade Federal de Viçosa (UFV). Professor Adjunto da UFT

\section{Adriano Firmino Valdevino de Araújo}

Graduação em Ciências Econômicas pela UFPB. Mestre e Doutor em Economia pela Universidade Federal de Pernambuco (UFPE). Professor Adjunto da UFT

\section{Aurélio Pessoa Picanço}

Engenheiro Sanitarista pela Universidade Federal do Pará (UFPA). Mestre e Doutor em Resíduos Sólidos pela Escola de Engenharia de São Carlos da Universidade de São Paulo (EESC-USP). Professor Adjunto da UFT

\section{Resumo}

O acesso aos serviços de saneamento ambiental, em especial, a coleta e a disposição final dos resíduos sólidos, é fundamental para a saúde pública e a preservação do meio ambiente, pois com a redução da má disposição do lixo, ocorre uma diminuição das endemias e da contaminação do solo. O investimento em saneamento ambiental é um dos grandes desafios para os gestores públicos, devido a sua ampla relação entre meio ambiente, saúde pública e desenvolvimento local. O presente trabalho visou estimar a máxima disposição a pagar pelo serviço de coleta de lixo para o município de Palmas, em Tocantins, por meio do método de avaliação contingente. Os resultados obtidos indicam que os principais fatores determinantes da disposição a pagar são: o nível de renda e a escolaridade.

Palavra-chave: políticas públicas; saneamento ambiental; método de avaliação contingente.

\section{Abstract}

The access to environmental sanitation services, especially the collection and final disposition of solid residues, is fundamental for the public health and environment preservation, because by reducing the bad garbage disposition, there is a decrease of the endemic diseases and soil contamination. The investment in environmental sanitation is one of the great challenges for public managers, due to its wide relationship among the environment, public health, and local development. The present work sought to esteem the willingness to pay for the garbage collection service for the municipal district of Palmas, in Tocantins, through the contingent evaluation method. The obtained results indicate that the main decisive factors of the willingness to pay are: the level of income and the education.

Keyword: public policies; sewage; contingent evaluation method. 


\section{Introdução}

A lei 11.445, de 05 de janeiro de 2007, que estabelece as diretrizes nacionais e o direcionamento das políticas públicas federais para o saneamento básico, apresenta no art. $2^{\circ}$ os princípios fundamentais que devem servir de base para os serviços públicos de saneamento básico. Um desses princípios fundamentais estabelece que o abastecimento de água, o esgotamento sanitário, a limpeza urbana e o manejo dos resíduos sólidos sejam realizados de forma adequada à saúde pública e à proteção do meio ambiente (BRASIL, 2007). De fato, há evidências que apontam uma estreita relação entre saneamento básico, saúde pública e meio ambiente. Mendonça e Motta (2005) afirmam que o déficit em saneamento gera consequências negativas à saúde da população, principalmente com as doenças transmitidas por vetores, contaminação do solo e poluição atmosférica. Teixeira e Pungirum (2005) corroboram o fato ao constatarem que as doenças registradas nos países em desenvolvimento decorrem da falta de saneamento. Segundo os dados da Fundação Nacional de Saúde FUNASA (2004), a falta de saneamento básico é responsável por $68 \%$ das internações em hospitais públicos.

Ainda segundo a FUNASA (2004) e IBGE (2004), para evitar a ocorrência de endemias, será preciso alcançar 100\% de cobertura do serviço de saneamento, remetendo a outro princípio fundamental estabelecido pela lei: a universalização do acesso (lei 11.445, art. $2^{\circ}$, inciso I). Entretanto, o cumprimento deste requer que recursos econômicos sejam destinados à ampliação e melhoria desses serviços. Estima-se que, para se atingir 100\% de cobertura dos serviços de saneamento, será preciso investir R 180 bilhões nos próximos anos (FUNASA, 2004).

Dado que os recursos econômicos são escassos, o maior investimento em saneamento básico implica no desvio de recursos que poderiam ser aplicados em outros setores, tais como: educação, segurança pública, redução da pobreza etc. Portanto, é imprescindível que as políticas públicas de saneamento básico sejam eficientes, de modo a atingir uma parcela maior da sociedade e, consequentemente, gerar maior benefício social. A eficiência e sustentabilidade econômicas dessas políticas constituem um dos princípios fundamentais do saneamento básico (BRASIL, 2007).

Um dos principais desafios dos centros urbanos deste século diz respeito ao correto manejo dos resíduos sólidos, pois a quantidade de lixo produzido e coletado é um indicador, tanto da saúde pública como da proteção ao meio ambiente.

De acordo com o Programa das Nações Unidas para o Desenvolvimento - PNUD (2001), a questão do saneamento básico no Tocantins é preocupante, pois além da inexistência de serviço de boa qualidade na maior parte dos municípios, a demanda por eles elevase em razão do fluxo migratório dirigido ao estado. Ainda, segundo o PNUD, o serviço de coleta de lixo nas cidades tocantinenses é deficitário em todas as etapas do processo de varrição, coleta e transporte do lixo, bem como na destinação final dos resíduos sólidos. Em Palmas,
Tocantins, a situação é um pouco melhor, de acordo com os dados do Sistema Nacional de Informação sobre Saneamento - SNIS (2004), o total de lixo urbano coletado no município é de 98,3\%, e 84\% da população tem o lixo coletado duas ou três vezes por semana.

Para Dias apud Junkes (2000), a geração do lixo é proporcional ao aumento da população e desproporcional à disponibilidade de soluções para o gerenciamento do distrito, resultando em sérias defasagens na prestação de serviços, o que causa uma diminuição do bem-estar. Ainda há a diferença do nível de serviços que o usuário deseja obter e daquele que ele está disposto a pagar. Segundo Carrera-Fernandez e Menezes (1999), na maioria dos municípios brasileiros não há a cobrança pelo serviço de coleta de lixo; assim, o custo do serviço de coleta e disposição do lixo é arcado integralmente pelo município.

Este trabalho teve por objetivo estimar o valor do benefício gerado a partir da melhoria do serviço de coleta e disposição do lixo na cidade de Palmas. Para tanto, foi utilizado o método de avaliação contingente (MAC) para a captação da disposição a pagar (DAP) dos indivíduos, a fim de que a melhoria seja implementada.

Além desta introdução, este trabalho é composto de quatro partes: na primeira parte é apresentada a metodologia; na segunda seção, a análise e a discussão dos resultados são apresentados; na terceira, é apresentada a conclusão e, por fim, as referências bibliográficas.

\section{Metodologia}

\section{0 método de avaliação contingente (MAC)}

A publicação do artigo de Cirray-Wantrup (1947) sobre a mensuração dos benefícios gerados pela preservação dos solos é um marco, na qual foram utilizadas, pela primeira vez, perguntas diretas à população para estimar o valor de um bem. Na década de 1960, Davis (1963) estimou a máxima DAP dos entrevistados pela área de recreação da floresta de Maine, nos Estados Unidos, a partir de perguntas diretas à população. No entanto, foi somente a partir da década de 1970 que a metodologia de avaliação contingente passou a ser mais explorada pelos economistas para avaliação de ativos ambientais. Segundo Maia (2004), a Envivonmental Protection Agency (EPA) financiou vários trabalhos que avaliaram as eficiências e deficiências do MAC, e isto foi fundamental para o desenvolvimento do método. Segundo CarreraFernandez e Menezes (1999), o MAC ganha sustentação metodológica a partir da formulação de Hannenann (1984), por meio de um modelo de maximização de utilidade e, por esse método, foi possível traduzir mudanças no nível de bem-estar dos indivíduos provenientes de bens e serviços não-ofertados em mercados formais, em valores monetários.

O MAC é utilizado na tomada de decisões ao definir políticas publicas e ambientais, e é o único método capaz de captar valores de existência para bens e serviços ambientais. O MAC utiliza um questionário para a obtenção de valores de bens para os quais não existe 
mercado. Com isso, cria-se um mercado hipotético, no qual o consumidor é contingenciado a respeito de um cenário apresentado, e neste são captadas as preferências do consumidor por um bem ou serviço ambiental. Quanto mais próximo às experiências reais for o cenário, mais preciso será o método. Mitchell e Carson (1989) apresentam três elementos no desenho do questionário para que o cenário se aproxime ao máximo do mercado real: i) a pesquisa deve ser constituída de uma descrição detalhada do bem; ii) é preciso definir algum tipo de questão na entrevista que revele a DAP do indivíduo do bem em questão e iii) é preciso também levantar outras variáveis socioeconômicas e as informações adicionais com relação ao problema em estudo.

Portanto, o MAC busca estimar o valor de um ativo a partir da mínima quantia que uma pessoa está disposta a receber, para ser compensada por um decréscimo na provisão de um bem (disposição a receber - DAR), ou da máxima quantia que uma pessoa estaria disposta a pagar para um incremento na provisão de um bem (DAP). A máxima (mínina) DAP (receber) é, muitas vezes, essencial para a construção de políticas públicas, pois revela as preferências das pessoas em relação aos recursos naturais.

Segundo o Painel da National Oceanic and Atmosphere Administration (NOAA) apud Maia et al. (2004), o formato referendo é recomendável para fazer uma avaliação contingente confiável. Este consiste em apresentar valores preestabelecidos aos entrevistados, os quais são indagados se aceitam, ou não, pagar (DAP) ou receber (DAR). Além de minimizar o comportamento estratégico, este formato reflete melhor a avaliação real do que se confrontasse com perguntas abertas para explicitar a DAP ou a DAR.

\section{Modelo econométrico}

Para se estimar a DAP pelos serviços de coleta e disposição de lixo, faz-se necessária a escolha de um modelo econométrico para tal. A abordagem desenvolvida por Hannenann (1984; 1989), e aplicada neste estudo, requer a utilização de um modelo econométrico em que a variável dependente é dicotômica ou binária. Um dos mais simples é o modelo de probabilidade linear (MPL). Porém, este modelo pode apresentar alguns problemas, sendo os principais: prever valores fora do intervalo $[0,1]$ e forte presença de heterocedasticidade. Usualmente, para a solução desses problemas, são utilizados os modelos logit ou probit ${ }^{1}$.

Apesar de apresentarem resultados bastante próximos, o modelo logit possui a vantagem de ter uma aplicação mais simples. A distribuição logística utilizada na estimação do modelo logit é algebricamente mais simples do que a distribuição normal, que é utilizada na estimação do probit. Por esse motivo, optou-se pela utilização do modelo logit para a estimação da DAP pelos serviços de água e esgoto².
O modelo logit parte de uma função logística de probabilidade acumulada, definida na Equação 1:

$\operatorname{Prob}\left(y_{i}=1\right)=\frac{e^{\beta^{\prime} X_{i}}}{1+e^{\beta^{\prime} X_{i}}}=\frac{1}{1+e^{-\beta^{\prime} X_{i}}}=F\left(\beta^{\prime} X_{i}\right)$

A variável $y_{i}$ representa uma dummy binária entre aceitar pagar ou não pelos serviços de coleta de lixo. X é o vetor de variáveis explicativas, enquanto b representa o vetor de parâmetros a serem estimados. Da mesma forma, pode-se definir na Equação 2:

$\operatorname{Prob}\left(y_{i}=0\right)=\frac{1}{1+e^{\beta^{\prime} X_{i}}}=1-F\left(\beta^{\prime} X_{i}\right)$

A esperança condicionada de $y_{i}$ é dada, portanto, por:

$$
\begin{gathered}
E\left(y_{i} / X_{i}\right)=0\left(\frac{1}{1+e^{\beta^{\prime} X_{i}}}\right)+1\left(\frac{e^{\beta^{\prime} X_{i}}}{1+e^{\beta^{\prime} X_{i}}}\right)=\frac{e^{\beta^{\prime} X_{i}}}{1+e^{\beta^{\prime} X_{i}}} \\
E\left(y_{i} / X_{i}\right)=\operatorname{Prob}\left(y_{i}=1\right)=F\left(\beta^{\prime} X_{i}\right)
\end{gathered}
$$

De acordo com a Equação 3, a função $\mathrm{F}\left(\mathrm{b}^{\prime} \mathrm{X}_{\mathrm{i}}\right.$ ) pode ser vista como a probabilidade condicional de $\mathrm{y}_{\mathrm{i}}$ assumir o valor 1 , dado um certo valor de b'X $X_{i}$, respeitando o intervalo $[0,1]$. Pela Equação 1, tem-se na Equação 4:

$$
\begin{array}{ll}
\lim _{\beta^{\prime} X_{i} \rightarrow \infty} & \operatorname{Prob}\left(y_{i}=1\right)=1 \\
\lim _{\beta^{\prime} X_{i} \rightarrow-\infty} & \operatorname{Prob}\left(y_{i}=1\right)=0
\end{array}
$$

A estimação do modelo logit pode ser feita a partir do método de máxima verossimilhança. Segundo Maddala (1983), a função de verossimilhança é definida como (Equação 5):

$$
\begin{gathered}
L=\prod_{y_{i}=1} F\left(\beta^{\prime} X_{i}\right) \prod_{y_{i}=0}\left[1-F\left(\beta^{\prime} X_{i}\right)\right] \\
L=\prod_{i=1}^{N}\left[\frac{e^{\beta^{\prime} X_{i}}}{1+e^{\beta^{\prime} X_{i}}}\right]^{y_{i}}\left[\frac{1}{1+e^{\beta^{\prime} X_{i}}}\right]^{1-y_{i}}
\end{gathered}
$$

A estimativa do vetor b deve maximizar essa função. O efeito da variação de uma das variáveis explicativas no valor esperado de $y_{i}$ é obtido derivando a Equação 3. Utilizando a Equação 1, o resultado dessa derivada pode ser escrito como na Equação 6:

$$
\frac{\partial E\left(y_{i} / X_{i}\right)}{\partial X_{k i}}=\frac{\partial F\left(\beta^{\prime} X_{i}\right)}{\partial X_{k i}}=\frac{e^{\beta^{\prime} X_{i}}}{\left(1+e^{\beta^{\prime} X_{i}}\right)^{2}} \beta_{k}
$$

A Equação 6 mostra o efeito marginal de $\mathrm{X}_{\mathrm{ki}}$ em $\mathrm{y}_{\mathrm{i}}$. De modo a facilitar a estimação da probabilidade condicional, são efetuados

'Uma hipótese fundamental dos modelos de regressão é que os erros tenham variância constante, indicando a homocedasticidade. A heterocedasticidade é a quebra dessa hipótese e o

${ }^{2}$ Segundo Araújo (2002), o uso da DAP é mais difundido em relação ao uso da DAR, devido ao fato de o primeiro ser uma medida mais conservadora e, portanto, confiável. 
alguns procedimentos matemáticos. Admitindo que $Z_{i}=\beta^{\prime} X_{i}$, as Equações 1 e 2 podem ser escritas como:

$$
\begin{gathered}
\operatorname{Prob}\left(y_{i}=1\right)=\frac{1}{1+e^{-\beta^{\prime} X_{i}}}=\frac{1}{1+e^{-Z_{i}}}=F\left(\beta^{\prime} X_{i}\right) \\
\operatorname{Prob}\left(y_{i}=0\right)=\frac{1}{1+e^{\beta^{\prime} X_{i}}}=\frac{1}{1+e^{Z_{i}}}=1-F\left(\beta^{\prime} X_{i}\right)
\end{gathered}
$$

Dividindo a Equação 7 pela 8, obtém-se:

$$
\frac{\operatorname{Pr} o b\left(y_{i}=1\right)}{\operatorname{Pr} o b\left(y_{i}=0\right)}=\frac{F\left(\beta^{\prime} X_{i}\right)}{1-F\left(\beta^{\prime} X_{i}\right)}=\frac{1+e^{z_{i}}}{1+e^{-Z_{i}}}=e^{z_{i}}
$$

A Equação 9 é conhecida como razão de probabilidade em favor de a dummy assumir o valor 1 . Tomando o logaritmo natural dessa equação e denotando o resultado como $\mathrm{L}_{\mathrm{i}}$, tem-se:

$L_{i}=\ln \left(\frac{F\left(\beta^{\prime} X_{i}\right)}{1-F\left(\beta^{\prime} X_{i}\right)}\right)=Z_{i}=\beta^{\prime} X_{i}$

Segundo Gujarati (2000), para fins de estimação, é considerado um componente aleatório de perturbação na Equação 10, de forma que:

$L_{i}=\ln \left(\frac{F\left(\beta^{\prime} X_{i}\right)}{1-F\left(\beta^{\prime} X_{i}\right)}\right)=Z_{i}=\beta^{\prime} X_{i}+\varepsilon_{i}$

Onde:

$\mathrm{e}_{\mathrm{i}}$ : é o termo de perturbação estocástica.

A Equação 11 representa o modelo logit propriamente dito. Uma vez estimado o vetor b, a estimativa da probabilidade condicionada pode ser obtida resolvendo a Equação 11 para $\mathrm{F}\left(\mathrm{b}^{\prime} \mathrm{X}_{\mathrm{i}}\right)$.

Um problema ressaltado por diversos autores em relação à estimação do modelo logit é a presença de heterocedasticidade ${ }^{3}$. No entanto, segundo Johnston e Dinardo (2001), transformações nas observações podem resolver esse problema.

\section{Pesquisa de campo}

Foram realizadas duas pesquisas de campo, sendo uma piloto e uma final. A pesquisa piloto utilizou a forma de eliciação de lances livres, com intuito de encontrar a média e os valores extremos da DAP e verificar se os indivíduos eram ou não indiferentes quanto ao prestador do serviço. Portanto, foram construídos dois tipos de questionários: o tipo I, no qual o governo era o ofertante, e o tipo II, em que uma empresa terceirizada era a ofertante. Foram aplicados, respectivamente, 50 e 54 questionários. Outro objetivo da pesquisa piloto foi testar os questionários e treinar os entrevistadores.
A pesquisa piloto foi realizada na cidade de Palmas, entre os dias 5 e 9 de maio de 2007, nos quais foi selecionado macroquadra com característica representativa da população; a pesquisa ficou concentrada em três partes: no Plano Diretor Norte, na qual foram aplicados 18 questionários; no Plano Diretor Sul, que teve maiores números de entrevistas devido a sua abrangência totalizando 54 questionários; e nos Aurenys, com 32 aplicações de questionários.

A pesquisa final foi determinada pelo critério de amostragem por conglomerados em múltiplos estágios. Neste desenho, subdivide-se inicialmente a população em setores e, em seguida, listam-se os domicílios dos setores sorteados. Esta listagem pode ser feita pelo nível de renda média dos setores e, após isto, procede-se à escolha dos domicílios a serem pesquisados em cada setor. Nesta pesquisa, a listagem dos setores foi determinada pela mediana da renda, e as escolhas dos domicílios selecionados por conglomerados foram realizadas por sorteio aleatório em cada quadra. A pesquisa final foi realizada em outubro e novembro de 2007 e o tamanho da amostra foi de 400 questionários para um nível de significância de 5\% e um erro absoluto de 5 .

Como forma de captação da DAP, utilizou-se o formato referendo com os valores dos lances compreendidos no intervalo de $\mathrm{R} \$ 1,00$ a $\mathrm{R} \$ 30,00$, e o peso de cada valor foi determinado pela frequência de ocorrência na pesquisa piloto, de acordo a Tabela $1^{4}$.

Os questionários foram aplicados nas quadras: 108 Norte, 305 Norte, 404 Norte, 409 Norte, 605 Norte, 1206 Sul, 207 Sul, 403 Sul, 606 Sul, 904 Sul, 906 Sul, totalizando 225 questionários aplicados no Plano Diretor. Nos setores periféricos, foram aplicados mais de 175 questionários, distribuídos entre os setores Aurenys e Taquaralto.

Para a realização da pesquisa de campo, foi ministrado um curso aos entrevistadores sobre técnicas de pesquisa de campo, com carga horária de 30 horas aulas. Após a realização do curso, foram selecionadas duas equipes compostas com quatro entrevistadores e um chefe de equipe. A escolha dos endereços nos conglomerados seguiu o critério da aleatoriedade e, no caso de não haver residente no endereço especificado, saltava-se uma casa.

Tabela 1 - Valores dos lances

\begin{tabular}{|ccc|} 
Valor do lance $(\mathrm{R} \$)$ & Frequência & Percentual \\
\hline 1,00 & 140 & 35,00 \\
\hline 2,00 & 37 & 9,25 \\
2,50 & 30 & 7,50 \\
\hline 4,00 & 12 & 3,00 \\
\hline 5,00 & 36 & 9,00 \\
10,00 & 91 & 22,75 \\
\hline 12,00 & 18 & 4,50 \\
\hline 20,00 & 24 & 6,00 \\
\hline 30,00 & 12 & 3,00 \\
\hline Total & 400 & 100,00 \\
\hline
\end{tabular}

Fonte: Elaboração própria a partir da pesquisa de campo. 
A confecção dos questionários seguiu os critérios utilizados em outros trabalhos na área (CARRERA-FERNANDEZ; MENEZES, 1999; LUCENA; TÁVORA JÚNIOR, 2000; ARAÚJO, 2002; PAIXÃO; MAIA FILHO, 2003). O questionário foi dividido em quatros partes: a primeira contendo questões socioeconômicas e demográficas; a segunda com questões específicas de saneamento; na terceira, questões sobre a DAP e, por fim, uma questão que trata da quantidade de bens duráveis que os indivíduos possuem. Isso teve como objetivo verificar se as respostas, da primeira parte, são coerentes com o padrão de vida dos entrevistados.

\section{Análise e discussão dos resultados}

\section{Descrição e análise da amostra}

Como resultado da pesquisa piloto, tem-se que a máxima DAP teve como valor mínimo não-nulo $\mathrm{R}$ \$ 1,00 e valor máximo de R\$ 30,00 . Outro resultado importante foi que os indivíduos se demonstraram indiferentes quanto ao prestador do serviço da coleta de lixo.

A pesquisa final foi realizada na cidade de Palmas, entre 12 de outubro a 16 de novembro de 2007. A análise dos dados foi efetuada pelo pacote estatístico STATA: Statistics/Data Analysis 9.2. Ao todo, foram aplicados 400 questionários, sendo que 68\% dos entrevistados eram mulheres e $32 \%$, homens. A idade média dos participantes da pesquisa foi de 38,45 anos, com desvio padrão de 13,70. Quanto à escolaridade, a média de estudo é de 9,49 anos, com desvio padrão de 4,19 anos; isto implica que os chefes de família de Palmas têm, em média, o Ensino Médio Incompleto. Por outra medida de tendência central, a mediana, a população de Palmas tem aproximadamente 11 anos de estudo, ou seja, o Ensino Médio Completo.

Tabela 2 - Composição da amostra segundo a ocupação dos indivíduos

\begin{tabular}{lll}
\hline Ocupação & Frequência & Percentual \\
\hline Aposentado pensionista & 22 & 5,73 \\
\hline Profissional liberal & 65 & 16,93 \\
\hline Desempregado & 127 & 33,07 \\
\hline Funcionário privado & 73 & 19,01 \\
\hline Funcionário público & 97 & 25,26 \\
\hline Total & 384 & 100 \\
\hline
\end{tabular}

Fonte: Resultado da pesquisa.
Quanto ao tipo de ocupação dos entrevistados, a maior parte deles declarou estar desempregado, com 33,07\% dos participantes da pesquisa e, em segundo lugar, estão os funcionários públicos com $25,26 \%$ da amostra, demonstrando que a participação do poder público é um fator determinante na formação da renda familiar dos indivíduos, seguido do setor privado, o qual representa 19,01\% da pesquisa e, em quarto lugar, com $16,93 \%$, os profissionais autônomos e liberais. Por fim, estão os aposentados e pensionistas, que representam 5,73\% dos entrevistados, como mostra a Tabela 2.

Como resultado da Tabela 3, tem-se que a renda média individual dos chefes de famílias é de $\mathrm{R} \$ 899,76$; isto equivale a 53\% da renda familiar que de $\mathrm{R} \$ 1.704,63$. Este resultado mostra a grande participação dos outros membros na composição da renda familiar. Outro dado importante é o desvio padrão da renda individual ( $R$ \$ 1.403,15) e familiar ( $\$$ \$ 1.923,63) e os valores máximos e mínimos da renda familiar que são, respectivamente, $\mathrm{R} \$ 15.000,00$ e $\mathrm{R} \$$ 250,00; quanto aos da renda individual são $\mathrm{R} \$ 10.000$ e $\mathrm{R} \$ 0,00$, respectivamente. Estes valores evidenciam uma forte desigualdade de renda da população de Palmas. Esta discrepância é explicada pela composição da amostra, pois os setores selecionados são heterogêneos entre si. Considerando a mediana, que é uma medida mais robusta de tendência central, as rendas familiar e individual são $\mathrm{R} \$ 900,00$ e R $\$ 400,00$

Considerando as condições de habitação, 65,08\% dos entrevistados declaram residir em casa própria, 29,10\% revelaram estarem habitados em casa alugada e 5,83\% declararam morar em casa emprestada ou outra. Os domicílios possuem em média 5,45 cômodos e 1,6 sanitários. Em relação ao serviço de coleta de lixo, os entrevistados demonstraram conhecer a sua importância, de forma que 73,44\% dos indivíduos que participaram da pesquisa apontaram alguma consequência danosa do lixo ao homem, somente 26,56\% não souberam relacionar as consequências do lixo.

Em se tratando do serviço de coleta de lixo, os domicílios pesquisados possuem este serviço. Em relação ao destino do lixo, $58,85 \%$ dos entrevistados não souberam qual era o destino do lixo da cidade, e dos 40,10\% que disseram conhecer o destino do resíduo sólido urbano, 64\% estão corretos, pois afirmaram que o destino é o aterro sanitário, sendo que 36\% estão equivocados quanto ao real destino do lixo.

Quando se trata da quantidade de lixo domiciliar produzida por ano, tem-se que, em média, 647,46 kg/ano são produzidos e com desvio padrão de $687,01 \mathrm{~kg} / \mathrm{ano}$. Isto equivale a uma produção per capita por dia de $0,53 \mathrm{~kg}$, com desvio padrão de 0,56 kg, esta

Tabela 3 - Estatística descritiva das rendas individual e familiar (em reais)

\begin{tabular}{|c|c|c|c|c|c|}
\hline & Média & Desvio padrão & Mediana & Valor mínimo & Valor máximo \\
\hline Renda individual & 899,76 & $1.403,15$ & 400 & 0 & 10.000 \\
\hline Renda familiar & $1.704,63$ & $1.923,63$ & 990 & 250 & 15.000 \\
\hline
\end{tabular}

Fonte: Resultado da pesquisa.

${ }^{5}$ Ver Azevedo (2008) 
quantidade está de acordo com a média de lixo de várias cidades brasileiras, correspondente a $0,5 \mathrm{~kg}^{5}$.

Quanto à DAP pelo serviço de coleta de lixo, 53,16\% dos chefes de famílias estão dispostos a pagar, enquanto 46,84\% dos chefes entrevistados não aceitam. Os principais motivos para rejeição em pagar são apresentados na Tabela 4, juntamente com as frequências absolutas e relativas de respondentes. O principal motivo da recusa pelo pagamento foi o entendimento que o governo deve se responsabilizar pela melhoria dos serviços de coleta e disposição do lixo, sem ônus adicional para o beneficiário. Tal motivo, juntamente com a justificativa "já pago muitos impostos", foi considerada como voto de protesto, caracterizando uma situação na qual o indivíduo está ciente dos benefícios da provisão do serviço, mas não quer pagar por este. Os votos de protesto representaram 47,73\% dos motivos da não-DAP e a outra causa de rejeição da DAP é o motivo financeiro equivalente à 42,05\% e, por fim, alguns entrevistados declaram que a obra não é de seu interesse - isto equivale a $10 \%$.

Para medir o nível certeza das respostas, foi utilizada uma escala percentual de valor, variando no intervalo de 0 a 100, o valor mínimo indica incerteza absoluta e o máximo representa certeza absoluta. Como resultado, obteve-se que as respostas dos indivíduos se concentraram mais nos níveis de certeza de 100, 50 e 80\% e que, em todos os níveis, o número de aceitação é maior do que o de rejeição. Isto demonstra que a população está mais ciente quanto ao real benefício da provisão do serviço de coleta de lixo.

\section{Estimação da DAP}

Para estimação do modelo, foram consideradas apenas as variáveis estatisticamente significativas a um nível de até 10\%, sendo excluídas, individualmente, as variáveis que tinham valor de p maior que $10 \%$. Em seguida, era novamente rodado o mesmo

Tabela 4 - Motivos de não estar DAP pelo serviço de coleta e disposição de lixo

\begin{tabular}{lcc} 
Motivos da não-DAP & Frequência & Percentual \\
\hline Obra não é de seu interesse & 18 & 10,23 \\
Motivos financeiros & 74 & 42,05 \\
Voto de protesto & 84 & 47,73 \\
Total & 176 & 100 \\
\hline
\end{tabular}

Fonte: Resultado da pesquisa. modelo, com exceção da variável excluída. Apesar de este procedimento ter sido realizado com e sem os votos de protesto, o modelo analisado neste estudo desconsidera as observações de protesto.

A Tabela 5 apresenta as estimativas dos modelos logit. Foram considerados quatro modelos. No modelo padrão, computou-se 1 para os aceites e 0 para as recusas, sem considerar o nível de certeza declarado pelos entrevistados. No modelo "Sim 80 a 100\%", foi computado 1 para os aceites com nível de certeza acima de $80 \%$ e 0 para as demais observações. Os modelos "Sim 90-100\%" e "Sim 100\%" seguiram o mesmo padrão, diferenciando apenas o nível de certeza associado ao aceite. O valor entre parênteses é o resultado da estatística $\mathrm{p}$, que mede a probabilidade exata do coeficiente ser igual a zero. As estimativas foram calculadas a partir do pacote estatístico STATA: Statistics/Data Analysis 9.2.

Pode-se observar que a variável valor do lance possui relação inversa com a variável dependente, ou seja, quanto maior o valor do lance, menor será a probabilidade de o indivíduo aceitar pagar. A variável conjunta renda versus estudo, que é o produto entre as variáveis "renda individual" e "anos de estudo", é positivamente correlacionada com a DAP, indicando que um aumento no produto renda versus estudo aumenta a probabilidade de aceitação do pagamento. Este resultado indica que uma família com maior nível de renda e com mais anos de estudo é mais propensa a pagar a DAP. Adicionalmente, evidencia-se um comportamento no qual uma menor renda individual pode ser compensada por uma maior escolaridade e vice-versa. A constante não tem sentido prático. $O$ valor do pseudo $R^{2}$ indica o grau de ajuste dos modelos.

A média/mediana e o intervalo de confiança da DAP foram calculados por meio do pacote estatístico STATA: Statistics/Data Analysis 9.2, para um nível de significância de 5\%. Neste procedimento, foi utilizado o método de Krinsky e Robb (JEANTY, 2008). Ressalta-se que o modelo "Sim 100\%" foi desconsiderado por apresentar baixo ajuste (pseudo $R^{2}$ de $7 \%$ )

$\mathrm{Na}$ Tabela 6, os valores médios da DAP, o intervalo de confiança da DAP e a estimação anual do valor do serviço de coleta e disposição de lixo estão apresentados. A média estimada para a logit foi de $\mathrm{R} \$ 11,62$, variando no intervalo de $\mathrm{R} \$ 9,39$ a $\mathrm{R} \$ 15,23$. Considerando o nível de certeza dos indivíduos de 80 a 100\%, a média estimada é $R$ \$ 7,50 no intervalo de $\mathrm{R} \$ 5,79$ a $\mathrm{R} \$$ 9,75 e para um nível de certeza dos agentes de 90 a 100\%, a média é

Tabela 5 - Mensuração da DAP pelo modelo logit, considerando o nível de certeza dos respondentes

\begin{tabular}{|c|c|c|c|c|}
\hline \multirow{2}{*}{ Variáveis } & \multirow{2}{*}{ Logit padrão } & \multicolumn{3}{|c|}{ Nível de certeza dos indivíduos } \\
\hline & & Sim $80-100 \%$ & Sim $90-100 \%$ & $\operatorname{Sim} 100 \%$ \\
\hline Valor do lance & $-0,14(0,00)$ & $-0,19(0,00)$ & $-0,20(0,00)$ & $-0,13(0,00)$ \\
\hline Renda versus estudo & $1,74 \mathrm{E}-05(0,04)$ & $2,36 \mathrm{E}-05(0,02)$ & $2,57 \mathrm{E}-05(0,00)$ & 5,06E-06 $(0,41)$ \\
\hline Constante & $1,36(0,00)$ & $1,01(0,00)$ & $0,82(0,00)$ & $-0,35(0,10)$ \\
\hline Pseudo $R^{2}$ & 0,14 & 0,19 & 0,20 & 0,07 \\
\hline Observações & 285 & 212 & 193 & 283 \\
\hline
\end{tabular}

Fonte: Elaboração própria a partir dos dados da pesquisa de campo. 
Tabela 6 - Estimação da média e média da DAP (em reais)

\begin{tabular}{lccc} 
Nível de certeza & Média/ Mediana & Intervalo de confiança da DAP & Estimação anual do serviço de coleta de lixo \\
Logit padrão & 11,62 & $9,39-15,23$ & $4.886 .953,68$ \\
80 a $100 \%$ & 7,5 & $5,79-9,75$ & $3.154 .230,00$ \\
90 a $100 \%$ & 6,52 & $4,79-8,66$ & $2.742 .077,28$ \\
\hline
\end{tabular}

Fonte: Resultado da pesquisa.

Tabela 7 - Custo do serviço de coleta de lixo domiciliar em Palmas, TO (em reais)

$\begin{array}{lcccc}\text { Ano } & 2002 & 2003 & 2004 & 2005 \\ \text { Custos } & 2.524 .327 & 2.325 .156 & 2.540 .661 & 3.050 .513\end{array}$

Fonte: Plano Plurianual 2002/2005, Balanços Gerais 2002/2005 apud Silva (2007).

$\mathrm{R} \$ 6,52$ variando no intervalo de $\mathrm{R} \$ 4,79$ a $\mathrm{R} \$ 8,66$. Este resultado foi superior ao encontrado por Lucena e Távora Júnior (2000), os quais aplicaram o MAC para estudar a importância do lixo para a qualidade ambiental em Recife, PE, chegando a uma estimativa de DAP mensal da ordem de $\mathrm{R} \$ 4,20$.

Ainda pela Tabela 6, tem-se que a estimação anual da DAP foi de $\mathrm{R} \$ 4.886 .953,68$, para o logit padrão, de $\mathrm{R} \$ 3.154 .230,00$ para o nível de certeza dos indivíduos de 80 a $100 \%$ e de $\mathrm{R} \$$ 2.742.077,28, para o nível de certeza dos entrevistados de 90 a $100 \%$. Foi obtido como proxy a estimação anual da DAP para o logit padrão e comparou-se com o custo do serviço de lixo, o qual, em 2005, foi de R \$3.050.513,00, conforme a Tabela 7. Pode-se observar que o excedente do consumidor é aproximadamente de $\mathrm{R} \$ 1.836 .440,68$. Este pode ser investido pelo gestor público em programas de coleta seletiva de lixo, os quais aumentam a duração dos aterros sanitários, geram novos empregos e diminuem o custo dos produtos reciclados, ou o excedente também pode ser investido em programas de educação ambiental.

\section{Conclusões}

Considerando que o objetivo desta pesquisa foi estimar o benefício gerado por uma melhoria no serviço de coleta e disposição final do lixo na cidade de Palmas, o MAC se mostrou adequado, gerando estimativas conservadoras devido ao uso da DAP em relação à DAR.

Ainda na pesquisa piloto, observou-se que os indivíduos são indiferentes em relação ao executor da melhoria em tal serviço (poder público ou setor privado), evidenciando o fato de que os indivíduos estão mais interessados no real benefício da provisão do serviço.

Em se tratando da pesquisa final, observou-se que 53,16\% dos entrevistados aceitaram pagar o valor do lance, enquanto 46,84\% dos entrevistados não estão dispostos a isso. O principal motivo das recusas foi o entendimento de que o serviço de coleta e disposição do lixo é somente de obrigação do governo e que já pagam muitos impostos, sendo que tais observações foram consideradas votos de protesto $(47,73 \%$ das recusas). Observou-se, também, um percentual significativo de recusas por motivos financeiros (42,05\% das recusas).
A partir do modelo estimado, notou-se que a probabilidade de aceitação do valor apresentado depende do valor do lance (relação inversa) e do produto entre renda individual e escolaridade (relação direta). A relação inversa entre o valor do lance e a probabilidade de aceitação indica um comportamento típico: quanto maior o preço de um bem, menor será a demanda por ele. A significância estatística da variável renda versus escolaridade indica que menor escolaridade pode ser compensada com maior renda e vice-versa.

Em relação ao nível de certeza, constatou-se que quanto maior for o intervalo considerado, maior será o valor da DAP estimada. A média/mediana para o logit padrão foi de $\mathrm{R} \$ 11,62$, já para um nível de certeza dos entrevistados, entre 80 a 100\%, a média/mediana foi de $\mathrm{R} \$ 7,50$, e se considerando o nível de certeza de 90 a 100\%, a DAP média foi igual $\mathrm{R} \$ 6,52$.

Os valores estimados anuais, agregados pelo número de domicílio da cidade de Palmas permitem um exercício de avaliação custobenefício de políticas públicas voltadas para a melhoria do serviço de coleta e disposição do lixo. Tomando o modelo logit padrão como referência, estimou-se que o ganho social de uma melhoria do serviço de coleta e disposição do lixo é da ordem de $\mathrm{R} \$ 4.886 .953,68$ por ano. Portanto, qualquer intervenção do poder público que resulte em melhoria desse serviço e custe menos que esse valor por ano resultará em um benefício líquido social positivo. Dado um nível de melhoria de serviço, a política pública será mais eficiente quanto maior for esse benefício líquido social.

Pode-se observar que, considerando o ano de 2005, o custo de manutenção desse serviço foi - superando apenas a estimativa de DAP anual agregada obtida a partir do modelo "Sim 90-100\%" - da ordem de R \$2.742.077,28. Ou seja, tendo como referência os modelos logit padrão e "Sim 80-100\%", constata-se que políticas públicas eficazes para a melhoria do serviço de coleta e disposição do lixo podem gerar benefícios líquidos para a sociedade.

Destaca-se que as estimativas obtidas neste estudo desconsideram os benefícios diretos, gerados, por exemplo, pela redução de gastos em saúde pública, resultantes da redução dos casos de endemias. Portanto, o benefício líquido efetivo gerado pela melhoria do serviço de coleta e disposição do lixo pode ser maior do que o apresentado neste estudo.

Por fim, apresentam-se duas sugestões de problemas que podem ser exploradas em estudos futuros: (i) a incorporação de estimativas relacionadas aos benefícios não-considerados no presente estudo, como a redução de gastos em saúde pública e (ii) a análise do custo-benefício de programa específicos de melhoria da coleta e disposição do lixo. 


\section{Referências}

ARAÚJO, A.F.V. Valoração Ambiental: uma aplicação do modelo logit para a avaliação monetária do jardim botânico da cidade de João Pessoa PB. 2002. 117f. Dissertação (Mestrado em Economia) - Universidade Federal de Pernambuco, Recife, 2002

AZEVEDO, J. Informações estatísticas dos resíduos sólidos urbanos no Brasil e em algumas das suas capitais. 2008. Disponível em: < http://www. portaldomeioambiente.org.br/JMA>. Acesso em: 25 de mar 2008.

BRASIL. Lei n. ${ }^{\circ} 11.445$, de 5 de janeiro de 2007. Estabelece diretrizes nacionais para o saneamento básico; altera as Leis nos 6.766 , de 19 de dezembro de 1979, 8.036, de 11 de maio de 1990, 8.666, de 21 de junho de 1993, 8.987, de 13 de fevereiro de 1995; revoga a Lei no 6.528, de 11 de maio de 1978; e dá outras providências. Diário Oficial [da] República Federativa do Brasil. Brasília, DF, 2007.

CARRERA-FERNANDEZ, J.; MENEZES, W.F.A avaliação contingente e a demanda por serviço público de coleta e disposição de lixo: uma análise a partir da região do Alto Subaé - Bahia. Revista Econômica do Nordeste, v. 30, n. Especial, p. 810-827, 1999.

CIRIACY-WANTRUP, S.V. Capital Returns from Soil Conservation Practices. Journal of Farm Economics, v. 29, n. 4, p. 1181-96, 1947.

DAVIS, R.K. The value of outdoor recreation: an economic study of the Maine woods. Ph. D. Thesis, Harvard University, 1963.

FUNDAÇÃO NACIONAL DE SAÚDE (FUNASA). Falta de saneamento é causa de 68\% das internações em hospitais públicos. 2004. Disponível em: <http://www.funasa.gov.br/Web\%20Funasa/not/not2004/not141. htm>. Acesso em: 08 jan. 2008.

GREENE, W.H. Econometric Analysis. $3^{\text {rd }}$ ed. New Jersey: Prentice Hall, 1997.

GUJARATI, D.N. Econometria básica. 3a ed. São Paulo: Makron Books, 2000. 846p.

HANEMANN, W.M. Welfare evaluations in contingent valuation experiments with discrete responses. American Journal of Agricultural Economics, v. 66, n.3, p. 332-341, 1984.

Welfare evaluations in contingent valuation experiment with discrete response data: reply. American Journal of Agricultural Economics, v. 71 n.4, p. 1057-1061, 1989

INSTITUTO BRASILEIRO DE GEOGRAFIA E ESTATÍSTICA (IBGE). Pesquisa Nacional de Saneamento Básico (PNSB). Abastecimento de água, esgotamento sanitário, drenagem urbana e limpeza urbana de lixo no Brasil. Rio de Janeiro, 2004.

JEANTY, P.W. Constructing Krinsky and Robb Confidence Intervals for Willingness to Pay (WTP) Measures Using STATA. 2008. Disponível em: <http://ideas.repec.org/p/boc/asug07/8.html>. Acesso em: 07 jul. 2008.
JOHNSTON, J.; DINARDO, J. Econometric Methods. $5^{\text {a }}$ ed. New York: McGraw-Hill Book Company, 2001.

JUNKES, M.B. Procedimentos para o aproveitamento de resíduo sólidos urbanos em municípios de pequeno porte. Dissertação de Mestrado Florianópolis: Universidade Federal de Santa Catarina, 2002.

LUCENA, L.F.L.; TÁVORA JÚNIOR, J.L.T. A importância do lixo para a qualidade ambiental - PE: uma análise por valoração contingente. 2000. Disponível em: <www.anpec.org.br/encontro2006/artigos/A06A183.pdf > . Acesso em: 08 jan. 2006.

MADDALA, G.S. Limited-Dependent and Qualitative Variables in Econometrics. New York: John Wiley \& Son, 1983. 401p.

MAIA, A.G.; ROMEIRO, A.R.; REYDON, B.P. Valoração de recursos ambientais: metodologia e recomendações. Texto para discussão. IE/ UNICAMP, Campinas, n. 116, março, 2004, p. 30-38

MAIA, J.A.F. Avaliação de Políticas Públicas: Introdução à demanda contingente. Revista Conjuntura e Planejamento, n. 124, p. 36-46, 2004.

MENDONÇA, M.J.; Cardoso; MOTTA, S.R. Saúde e Saneamento no Brasil. IPEA, 2005. Texto para discussão, 1081.

MITCHELL, R.; CARSON, R. Using surveys to value publics goods: the contingent valuation method. Washington, D. C: Resources for the future, 1989. 463p.

PAIXAO, A.; MAIA FILHO, P. Estimação da Disposição a Pagar Pelos Serviços de Abastecimento de Água e Esgotamento Sanitário de João Pessoa - PB. Utilizando o Método de Avaliação Contingente. Revista Econômica do Nordeste, v. 34, n. 4, p. 574-588, 2003

PROGRAMA DAS NAÇÕES UNIDAS PARA O DESENVOLVIMENTO - PNUD. Relatório de Desenvolvimento Humano 2006. A água para lá da escassez: poder, pobreza e crise mundial da água. Disponível em: $<$ http://www.pnud.org.br/arquivos/rdh/rdh2006/rdh2006.zip>. Acesso em 21 março 2008.

SILVA, M.C. Investigação Sobre a Eficiência de Coleta de Lixo em Palmas-TO. 2007, 53 páginas. TCC (Bacharel em ciência Econômicas) Universidade Federal do Tocantins, Palmas, 2007.

SISTEMA NACIONAL DE INFORMAÇÕES SOBRE SANEAMENTO (SNIS): Diagnóstico do Manejo de Resíduos Sólidos Urbanos - 2004. Disponível em: <http://www.snis.gov.br/>. Acesso em: 08 jan. 2006.

TEIXEIRA, J.C.; PUNGIRUM, M.E.M.C. Análise da Associação entre Saneamento e Saúde nos Países da América Latina e do Caribe empregando dados secundários do banco de dados da Organização Pan-Americana de Saúde - OPAS. Revista Brasileira Epidemiologia, v. 8, n. 4, p. 365-376, 2005. 